Article

\title{
Development of Thermo-Responsive Polycaprolactone-Polydimethylsiloxane Shrinkable Nanofibre Mesh
}

\author{
Chia-Hsuan Hsieh ${ }^{1,+}{ }^{,}$Nur Adila Mohd Razali ${ }^{1,+}+\mathbb{C}$, Wei-Chih Lin $1, * \mathbb{C}$, Zhi-Wei Yu ${ }^{1}$, \\ Dwita Istiqomah ${ }^{1}$ (D), Yohei Kotsuchibashi ${ }^{2}$ and Hsing-Hao $\mathrm{Su}^{3, *(\mathbb{D}}$ \\ 1 Department of Mechanical and Electro-mechanical Engineering, National Sun Yat-sen University, \\ Kaohsiung 80424, Taiwan; B053021005@student.nsysu.edu.tw (C.-H.H.); \\ d063020007@student.nsysu.edu.tw (N.A.M.R.); m073020065@student.nsysu.edu.tw (Z.-W.Y.); \\ m083020087@student.nsysu.edu.tw (D.I.) \\ 2 Department of Materials and Life Science, Shizuoka Institute of Science and Technology, Shizuoka 437-8555, \\ Japan; kotsuchibashi.yohei@sist.ac.jp \\ 3 Department of Otorhinolaryngology, Kaohsiung Veterans General Hospital, Kaohsiung 81362, Taiwan \\ * Correspondence: wc.lin@mail.nsysu.edu.tw (W.-C.L.); shsu@vghks.gov.tw (H.-H.S.); \\ Tel.: +886-7-5252-000 (ext. 4226) (W.-C.L.) \\ $+\quad$ C.-H.H. and N.A.M.R. are the co-first authors with an alphabetical order.
}

Received: 15 June 2020; Accepted: 17 July 2020; Published: 21 July 2020

\begin{abstract}
A thermally activated shape memory polymer based on the mixture of polycaprolactone (PCL) and polydimethylsiloxane (PDMS) was fabricated into the nanofibre mesh using the electrospinning process. The added percentages of the PDMS segment in the PCL-based polymer influenced the mechanical properties. Polycaprolactone serves as a switching segment to adjust the melting temperature of the shape memory electro-spun PCL-PDMS scaffolds to our body temperature at around $37^{\circ} \mathrm{C}$. Three electro-spun PCL-PDMS copolymer nanofibre samples, including $\mathrm{PCL}_{6}-\mathrm{PDMS}_{4}, \mathrm{PCL}_{7}-\mathrm{PDMS}_{3}$ and $\mathrm{PCL}_{8}-\mathrm{PDMS}_{2}$, were characterised to study the thermal and mechanical properties along with the shape memory responses. The results from the experiment showed that the PCL switching segment ratio determines the crystallinity of the copolymer nanofibres, where a higher PCL ratio results in a higher degree of crystallinity. In contrast, the results showed that the mechanical properties of the copolymer samples decreased with the PCL composition ratio. After five thermomechanical cycles, the fabricated copolymer nanofibres exhibited excellent shape memory properties with $98 \%$ shape fixity and above $100 \%$ recovery ratio. Moreover, biological experiments were applied to evaluate the biocompatibility of the fabricated PCL-PDMS nanofibre mesh. Owing to the thermally activated shape memory performance, the electro-spun PCL-PDMS fibrous mesh has a high potential for biomedical applications such as medical shrinkable tubing and wire.
\end{abstract}

Keywords: shape memory polymer; nanofibres; electrospinning; thermo-responsive

\section{Introduction}

During a medical surgery, the knot-tying skill plays a vital role in the typical surgical suture, as the manipulation of the tying force depends on the medical operators. A tight suture treatment can lead to formation of a bean-like scar. Similarly, the dermis layer would be incompletely fixed which could result in overgrowth of fibroblasts in the wound gap and create scars. The research team developed a smart shape memory surgical suture which is made of polyurethane, and this biodegradable suture contains a tuneable shape property which can be controlled by changing the 
temperature [1]. Besides, recent research has also shown that the shape memory polymer- (SMPs) fabricated surgical sutures could provide a steady and uniform restoring force lasting more than two weeks for forming new tissue [2]. Temperature-responsive SMPs are smart materials that could be modulated to a temporary shape and can gradually recovered to their permanent shape upon exposure to heat $[3,4]$. Shape memory polymers have drawn great attention for their diverse biomedical applications such as smart sutures [1], drug deliveries [5] and cardiovascular stents [6].

Polycaprolactone (PCL)-based SMPs received much attention due to the fact of its various advantages such as biocompatibility, biodegradability and elasticity. Polycaprolactone (PCL) is useful as a switching segment for SMPs, as its melting temperature of PCL serves as the transition temperature which can be adjusted from 45 to $60^{\circ} \mathrm{C}$ with increasing $\mathrm{M}_{\mathrm{n}}$ [3], and this range is useful for deployment in vivo. The switching segment and soft segment are important criteria of SMP due to the fact of their dissimilarity in the structure and thermodynamic incompatibility. Besides PCL, PDMS is also widely used due to the fact of its remarkable thermal properties. Polydimethylsiloxane (PDMS) has an extremely low glass transition temperature $\left(T_{g}=-125^{\circ} \mathrm{C}\right)$ which makes PDMS an effective soft segment that can adjust the mechanical properties of PCL-based SMPs. It is mainly used for biomedical and food applications, as it are a non-toxic polymer. In general, PDMS has high performance, for example, in biocompatibility, low viscosity, good thermal properties, and chemical stability.

Shape memory polymer nanofibres have high potential for application as surgical sutures due to the face of their mechanical performance. As a result of its simple and robust performance, electrospinning has been a key choice to fabricate nanofibres that could possibly mimic the extracellular matrix (ECM) of the cells [7]. Extensive research has been done utilising the electrospinning technique to fabricate nanofibre from a wide variety of materials and polymers with different nanofibre structures and constructions. Mechanical properties of the SMP were affected by the different ratios of the switching segment and soft segment. For example, Zhang et al. [3] used PDMS with different molecular weights to control the chain length of the switching segment, and the results showed that the smaller molecular weight of PDMS could make better polymer with a higher shape recovery ratio and yield stress. Meanwhile, it is reported that the molecular weight of hard segment could form a stronger polymer with better shape recovery ratio $[5,8]$.

In this study, we synthesized a series of temperature-responsive PCL-PDMS nanofibres with different mixture ratios of PCL and PDMS. The PCL-PDMS raw material was synthesized from the polycaprolactone diol (PCL diol) and polydimethylsiloxane diol (PDMS diol) and was then utilised to fabricate the PCL-PDMS nanofibres samples through the electrospinning process. The addition of PDMS segment can enhance the deformability (i.e., \% strain at break) which can benefit the stretchability and flexibility of the SMPs. A series of experiments were conducted to characterise the thermal, mechanical and biocompatibility of the fabricated PCL-PDMS copolymer nanofibre.

\section{Materials and Methods}

This section describes the fabrication and characterisation of PCL-PDMS copolymer. In brief, the copolymers were synthesized and transformed into nanofibres structure via a conventional electrospinning process. Then, the fabricated copolymer nanofibres were assessed based on the chemical structure, surface morphology, thermal, mechanical and shape memory performance. The following subsection provides a detailed description of the procedures conducted for the assessment.

\subsection{Synthesis of PCL-PDMS Copolymer}

The PCL-PDMS copolymer was synthesized based on the protocol mentioned in $[9,10]$. The polyurethane synthesis was performed using polycaprolactone diol (PCL diol, $\mathrm{M}_{\mathrm{n}} \sim 2000$, Sigma-Aldrich, St. Louis, MO, USA) and dihydroxyl-terminated polydimethylsiloxane diol (PDMS diol, $\mathrm{M}_{\mathrm{n}} \sim 4200$, Sigma-Aldrich, St. Louis, MO, USA) at 6:4, 7:3 and 8:2 PCL-PDMS weight ratios. In this reaction, the PCL diol was regarded as a switching segment with a permanent shape property, while the PDMS diol was regarded as a soft segment with temporary shape property. In brief, different 
PCL and PDMS weight ratios were dissolved in $100 \mathrm{~mL}$ anhydrous toluene (99.8\%, Sigma-Aldrich, St. Louis, MO, USA) at $60{ }^{\circ} \mathrm{C}$ in a round-bottomed flask, and $1.16 \mathrm{~mL}$ of hexamethylene diisocyanate (HDI, Alfa Aesar, Ward Hill, MA, USA) cross-linker was added equivalent to the reactive hydroxyl groups in the solution. Subsequently, $8 \mathrm{mg}$ dibutyltin dilaurate (MSMD, ACROS Organics, Morris Plains, NJ, USA) catalyst was added into the flask. Then, the mixture was heated at $110{ }^{\circ} \mathrm{C}$ for $24 \mathrm{~h}$ under nitrogen atmosphere. N-hexane (99\%, Sigma-Aldrich) was used as the solvent for precipitation of the resultant copolymer. Figure 1 shows the synthetic procedure of the PCL-PDMS block copolymer.
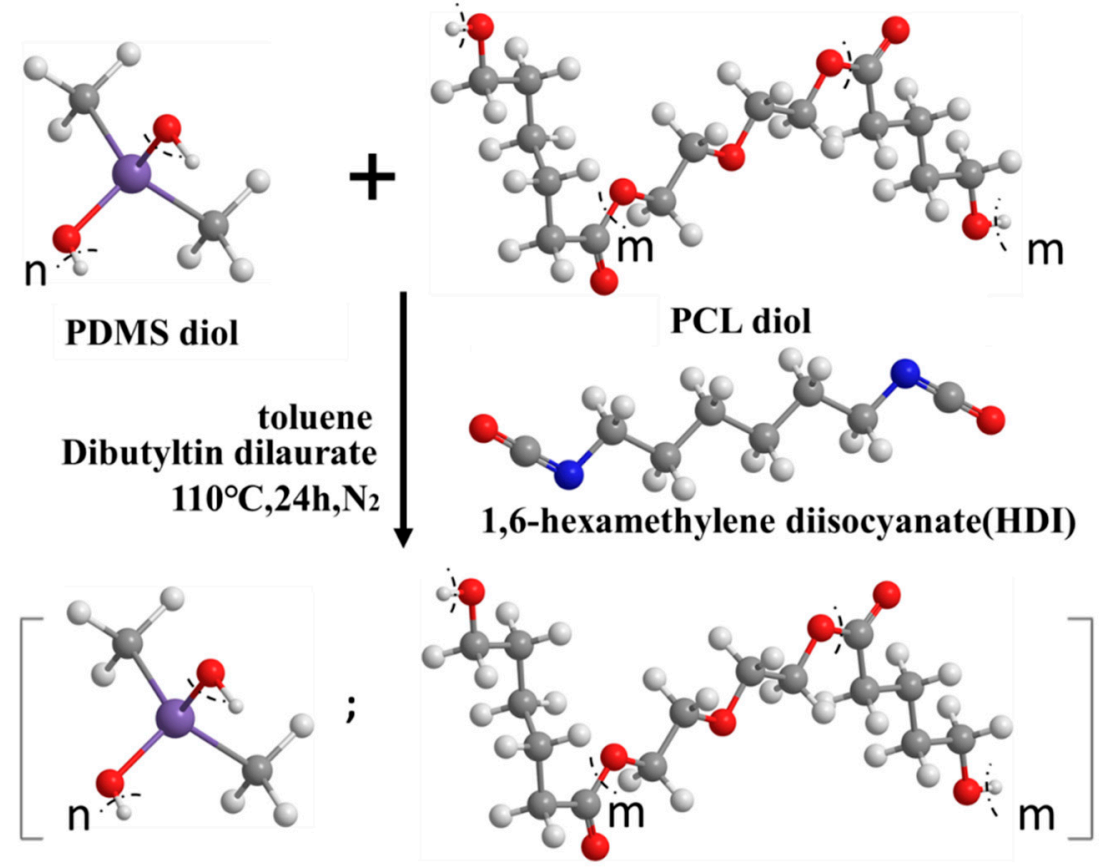

Poly(PCL/PDMS urethane)

Figure 1. The synthetic procedure to fabricate the polycaprolactone-polydimethylsiloxane (PCL-PDMS) copolymer.

\subsection{Fabrication of PCL-PDMS Copolymer Nanofibres}

The PCL-PDMS copolymer solutions were prepared at $10 \mathrm{wt} \%$ with 1,1,1,3,3,3-hexafluoro-2-propanol (HFIP, 99\%, Sigma-Aldrich) as solvent. The solutions were stirred overnight until homogeneous solutions were obtained. Then, the solutions were transferred to the electrospinning process to create electro-spun nanofibres. Each solution was transferred into a $3 \mathrm{~mL}$ syringe with a 24 gauge needle (diameter $\sim 0.26 \mathrm{~mm}$ ). The electrospinning process parameters were set at $15 \mathrm{kV}$ applied voltage, flow rate at $0.225 \mathrm{~mL} / \mathrm{h}$, and the distance between needle and collector was kept at $19 \mathrm{~cm}$. The solution was electro-spun on an aluminium foil and the fabricated nanofibres were dried in vacuum desiccator subjected to characterisation.

\subsection{Characterisation of the PCL-PDMS Copolymer Nanofibres}

\subsubsection{Nuclear Magnetic Resonance}

To verify the chemical structure of the PCL-PDMS copolymer nanofibres, proton nuclear magnetic resonance, 1H NMR (JEOL ECZ600R, Tokyo, Japan) was utilised. The samples were prepared by mixing $3 \mathrm{mg}$ of the copolymer nanofibres with $3 \mathrm{~mL}$ of chloroform-d (CDCL3, Sigma-Aldrich) solvent. The 1H NMR spectra were recorded at $400 \mathrm{MHz}$, at room temperature. Each sample was scanned 16 times to obtain the average spectrum. The chemical shifts corresponded relatively to the solvent at 7.3 ppm in 1H NMR spectra. 


\subsubsection{Surface Morphology of the PCL-PDMS Copolymer Nanofibres}

Scanning electron microscopy (SEM, JSM630, JEOL, Tokyo, Japan) was utilised to observe the fabricated nanofibres' morphology including the size, shape and fibre diameter. Prior to SEM scanning, the samples were sputtered with gold and observed microscopically at an accelerating voltage of $10 \mathrm{kV}$. The nanofibres' diameter was analysed manually from the SEM images using ImageJ software (version 1.44, National Institutes of Health, Bethesda, Maryland, USA). At least 200 diameters were randomly selected for each sample and the average nanofibre diameter was calculated.

\subsubsection{Thermal Properties of PCL-PDMS Copolymer}

Differential scanning calorimetry (DSC, DSC-60 Plus, Shimadzu, Kyoto, Japan) was performed to assess the thermal properties of the copolymer including the degree of crystallinity and the melting temperature. The samples were sealed in perforated aluminium and the test temperature was set from $0{ }^{\circ} \mathrm{C}$ to $150^{\circ} \mathrm{C}$ under a nitrogen $(50 \mathrm{~mL} / \mathrm{min})$ environment at $10{ }^{\circ} \mathrm{C} / \mathrm{min}$ heating rate. The melting point $\left(\mathrm{T}_{\mathrm{m}}\right)$ was determined by the peak of heat flow, and the crystallinity $\left(X_{c}\right)$ was calculated using the following equation:

$$
\% X_{c}=\frac{\Delta H_{m}}{\Delta H_{m}^{0}} \times 100
$$

where $\Delta H_{m}$ is normalised based on the $\%$ mass of PCL segments in the fibre $139.5 \mathrm{~J} / \mathrm{g}$ for $100 \%$ crystalline PCL [11].

\subsubsection{Mechanical Properties of PCL-PDMS Copolymer Nanofibres}

The ultimate tensile strength of the fabricated nanofibres was evaluated using a uniaxial tensile tester (FGP-0.5, Nidec-SHIMPO, Kyoto, Japan) at a rate of $20 \mathrm{~mm} / \mathrm{min}$ at ambient temperature until failure. The samples were cut into a dimension of $30 \mathrm{~mm} \times 5 \mathrm{~mm} \times 100 \mu \mathrm{m}$ (length $\times$ width $\times$ thickness) and clamped for uniaxial tensile test. Tensile strength, percentage elongation and Young's modulus were determined from the linear region of the resultant stress-strain curve. The measurements were replicated five times.

\subsubsection{Shape Memory Behaviour}

The shape memory behaviour of the copolymer samples were evaluated using strain-controlled cyclic thermomechanical tensile tests [3]. Rectangular samples with a dimension of $30 \mathrm{~mm} \times 5 \mathrm{~mm} \times$ $100 \mu \mathrm{m}$ (length $\times$ width $\times$ thickness) were prepared and tested to the following processes sequentially. The shape memory effect was quantitatively measured as follows: (a) the samples were equilibrated at $40{ }^{\circ} \mathrm{C}$ for $10 \mathrm{~min}$ and stretched to $50 \%$ strain at a rate of $20 \mathrm{~mm} / \mathrm{min}\left(\varepsilon_{m}=50 \%\right)$, (b) cooled to room temperature $\left(25^{\circ} \mathrm{C}\right)$ with an isothermal time of $3 \mathrm{~min}$ to fix the temporary shape, (c) unloaded to $0.01 \mathrm{~N}$ at a rate of $20 \mathrm{~mm} / \mathrm{min}$ and measured the strain $\left(\varepsilon_{f}\right)$, and (d) re-heated the samples to $40{ }^{\circ} \mathrm{C}$ to recover the permanent shape and measured the strain $\left(\varepsilon_{r}\right)$. The processes were repeated for five cycles $(N)$ for multiple cycle effects. The shape memory performances of the material were evaluated based on the ability of the material to maintain mechanical deformation after cooling for temporary deformation (shape fixity ratio, $R_{f}$ ) and to return to its permanent shape after mechanical deformation (shape recovery ratio, $R_{r}$ ) and as given [12]:

$$
\begin{gathered}
R_{f}(N)=\frac{\varepsilon_{f}(N)}{\varepsilon_{m}} \times 100 \% \\
R_{r}(N)=\frac{\varepsilon_{m}-\varepsilon_{r}(N)}{\varepsilon_{m}-\varepsilon_{r}(N-1)} \times 100 \%
\end{gathered}
$$

where $\varepsilon_{r}(N)$ and $\varepsilon_{r}(N-1)$ are the final strains in two successive cycle in the stress-free state during the recovery process, and $\varepsilon_{f}(N)$ is the ultimate strain in the stress-free state in the fixing process. 


\subsection{Cytotoxicity and Biocompatibility Study}

Cell cytotoxicity and biocompatibility of the synthesized copolymer nanofibres were evaluated using primary dermal fibroblast (HDF) from American Type Culture Collection (ATCC). The HDF cells were cultured in Dulbecco's modified Eagle's medium (DMEM, Hyclone) supplemented with $10 \%$ foetal bovine serum (FBS, Hyclone) and $1 \%$ antibiotic-antimycotic (AAS, Hyclone) at $37{ }^{\circ} \mathrm{C}$ in $5 \%$ carbon dioxide $\left(\mathrm{CO}_{2}\right)$ environment until it reached $80 \%$ confluency. Before seeding, the nanofibres were fixed in 24 well culture plates and sterilized under UV light for $2 \mathrm{~h}$. The HDF cells were seeded at a density of $5 \times 104$ per each well, respectively. The culture plates were incubated at $37^{\circ} \mathrm{C}$ with $\% \mathrm{CO}_{2}$ and the culture medium was changed every other day.

The viability of the cells cultured on the nanofibres sample was monitored using Cell Counting Kit-8 (CCK-8, Sigma-Aldrich) assay according to the manufacturer instructions on day 1 and day 3 . At each time point, $100 \mu \mathrm{L} \mathrm{CCK}-8$ solutions in $1 \mathrm{~mL}$ culture medium was added into each well and further incubated for another $2 \mathrm{~h}$. The absorbance was measured at $450 \mathrm{~nm}$ using a microplate reader (BIO-RAD Model 550, Tokyo, Japan). In addition, the biocompatibility of the material was observed through dual staining of fluorescein diacetate (FDA, Sigma-Aldrich, St. Louis, MO, USA)/DAPI, (4',6-diamidino-2-phenylindole, Sigma-Aldrich, St. Louis, MO, USA). The HDF cells were cultured on the sterilized samples and at specific time intervals, the cultured cells were washed with PBS and stained with FDA $(5 \mu \mathrm{g} / \mathrm{mL}$, working concentration) and DAPI $(0.1 \mu \mathrm{g} / \mathrm{mL}$, working concentration) for $15 \mathrm{~min}$ to display live cells observed under a fluorescent microscope. Live cell morphology was observed with an inverted fluorescence microscope (Eclipse TS2, Nikon, Tokyo, Japan).

\section{Results and Discussion}

\subsection{Molecular Characteristic of PCL-PDMS Copolymer Nanofibres by NMR}

The molecular characteristics of the fabricated PCL-PDMS copolymer nanofibres with different ratios were investigated using $1 \mathrm{H}$ NMR spectroscopy. Since all of the samples have similar peaks, only the spectrum for $\mathrm{PCL}_{6}-\mathrm{PDMS}_{4}$ is presented. Figure 2 shows the $1 \mathrm{H}$ NMR spectra for the $\mathrm{PCL}_{6}-\mathrm{PDMS}_{4}$ sample and the proton signals associated with both PCL and PDMS are observed. For the PCL segment, the peak detected at $1.63 \mathrm{ppm}$ is attributed to the $-(\mathrm{CH} 2) 3-, 2.30 \mathrm{ppm}$ for $-\mathrm{CH} 2 \mathrm{CO}-$ and at $4.04 \mathrm{ppm}$ for -OCH2- (methylene protons alpha to the ester group of PCL segments). Meanwhile, methylene protons in the repeated units of PDMS segments were observed at $0.05 \mathrm{ppm}$ peak. The copolymer composition and the molecular weight of all copolymers were determined at $0.05 \mathrm{ppm}$ (PDMS) and $4.04 \mathrm{ppm}$ (PCL). Table 1 summarised the molecular properties of the PCL-PDMS copolymer nanofibres. Based on the calculated data, the weight ratio of the PCL and PDMS in all copolymer compositions was found to be consistent with the feed ratio, thus, confirming the successful fabrication of the PCL-PDMS copolymer nanofibres.

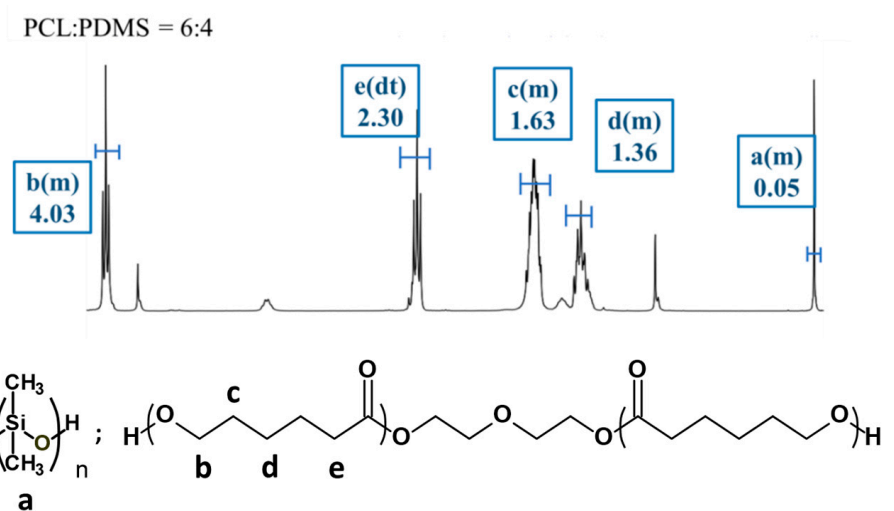

Figure 2. Compositional analysis of the PCL-PDMS copolymer nanofibres by ${ }^{1} \mathrm{H}$ NMR spectroscopy. 
Table 1. Compositional analysis of the PCL-PDMS copolymer nanofibres by 1H NMR spectroscopy.

\begin{tabular}{cccccc}
\hline \multirow{2}{*}{ Sample } & \multicolumn{2}{c}{ Feed Ratio (wt $\%)$} & \multicolumn{2}{c}{ Composition $\mathbf{( w t} \%)$} & \multirow{2}{*}{$\mathbf{M}_{\mathbf{n}}\left(\times \mathbf{1 0}^{\mathbf{3}}\right)$} \\
\cline { 2 - 4 } & PCL & PDMS & PCL & PDMS & \\
\hline PCL $_{6}-\mathrm{PDMS}_{4}$ & 60 & 40 & 61.5 & 38.5 & 82.74 \\
PCL $_{7}-\mathrm{PDMS}_{3}$ & 70 & 30 & 69.6 & 30.4 & 37.00 \\
PCL $_{8}-\mathrm{PDMS}_{2}$ & 80 & 20 & 83.5 & 16.5 & 25.95 \\
\hline
\end{tabular}

\subsection{PCL-PDMS Copolymer Nanofibres' Surface Morphology}

The PCL-PDMS copolymers were fabricated into nanofibre mesh via the conventional electrospinning process. The copolymer solutions were prepared at $10 \mathrm{wt} \%(\mathrm{w} / \mathrm{w})$ diluted in HFIP and electro-spun at $10 \mathrm{kV}$. In general, with an appropriate polymer concentration, polymers with $M_{n}>10,000$ will form continuous fibres during the electrospinning process [13]. Referring to Table 1, all the synthesized PCL-PDMS copolymer demonstrated $M_{n}>10,000$ which is within the expected electrospinning fibre-forming range. Figure 3 illustrates the SEM images of the fabricated nanofibres from different copolymer composition ratios. The surface morphology of PCL-PDMS copolymer nanofibres show a continuous, smooth and bead-free structure with a random nanofibre orientation.
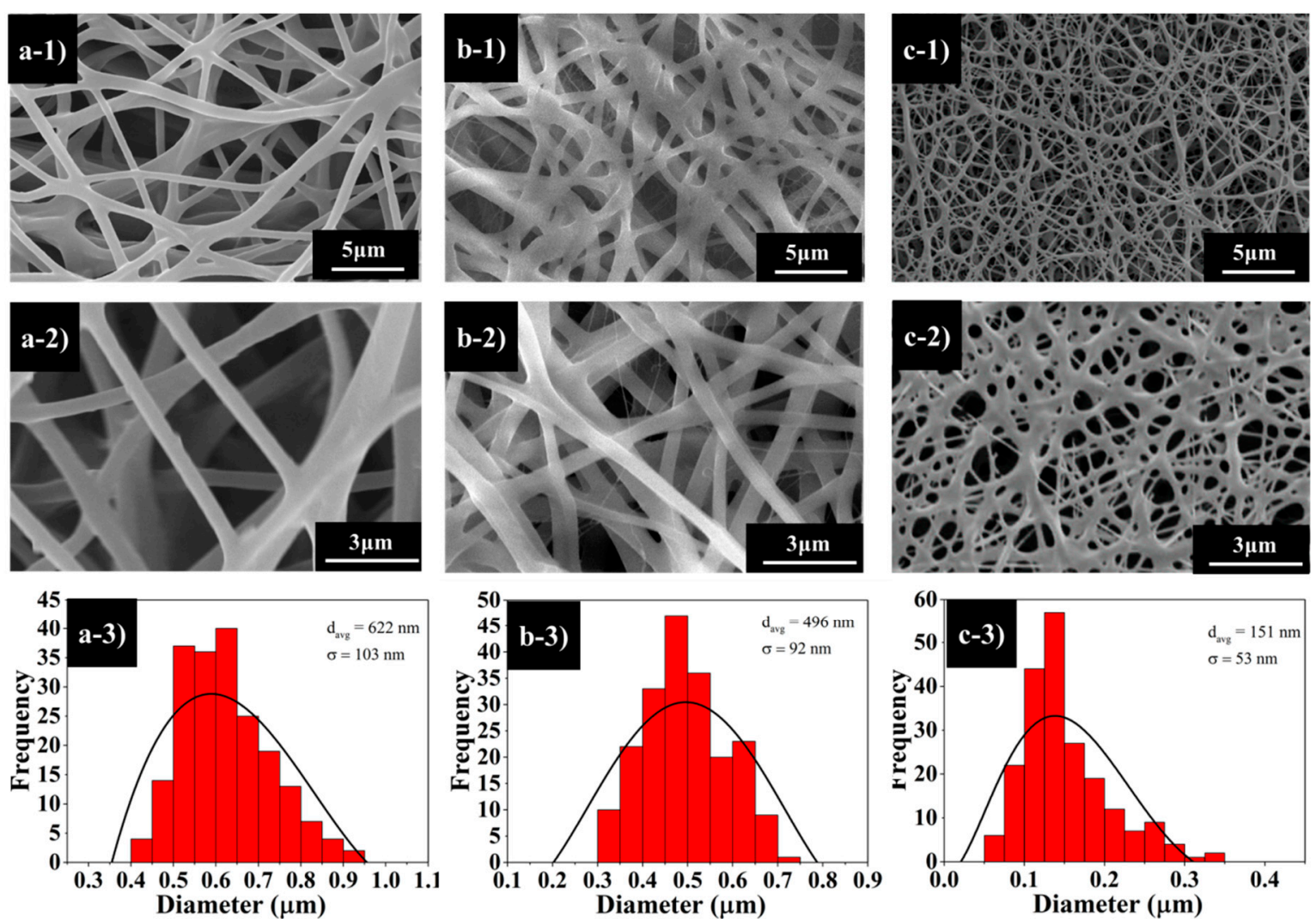

Figure 3. The scanning electron microscope (SEM) images of electro-spun PCL-PDMS copolymer nanofibres: (a-1,a-2,a-3) $\mathrm{PCL}_{6}-\mathrm{PDMS}_{4} ;(\mathbf{b}-\mathbf{1}, \mathbf{b}-\mathbf{2}, \mathbf{b}-\mathbf{3}) \mathrm{PCL}_{7}-\mathrm{PDMS}_{3} ;(\mathbf{c}-\mathbf{1 , c - 2 , c - 3}) \mathrm{PCL}_{8}-\mathrm{PDMS}_{2}$.

The $\mathrm{PCL}_{6}-\mathrm{PDMS}_{4}$ (Figure 3a) and $\mathrm{PCL}_{7}-\mathrm{PDMS}_{3}$ (Figure 3b) samples showed cylindrical and uniform nanofibres; meanwhile, the $\mathrm{PCL}_{8}-\mathrm{PDMS}_{2}$ sample showed a melted fibrous morphology. This might be related to the number-average molecular weight $\left(\mathrm{M}_{\mathrm{n}}\right)$ of the synthesized copolymer as obtained from the NMR spectroscopy (Table 1). The molecular weight of the synthesized copolymers decreased with the decreases of the PDMS mixture ratio. The melt fibrous morphology of the $\mathrm{PCL}_{8}-\mathrm{PDMS}_{2}$ sample indicated an insufficient physical cross-linking with less interconnected junction formed among the fibres. The average diameter of the copolymer nanofibres ranged from $151 \mathrm{~nm}$ to 
$622 \mathrm{~nm}$ when the PDMS composition increased from 20\% to 40\%. A larger nanofibre diameter was achieved with a sample with higher PDMS compositions. The increase in resultant nanofibre diameter was attributed to the molecular weight $\left(M_{n}\right)$ of the sample, where higher $M_{n}$ will increase the fibre diameter due to the high number of chain entanglement and increased viscosity.

\subsection{Thermal Properties of the PCL-PDMS Copolymers}

The thermal properties of the copolymer samples were characterised by DSC. The DSC curves after the second heating run were illustrated in Figure 4. The measurement was made after the second heating run to remove residual solvent and to erase the thermal history of the polymer during the synthesis process. It can be observed that the melting temperatures $\left(\mathrm{T}_{\mathrm{m}}\right)$ for all PCL-PDMS compositions were similar-approximately $37^{\circ} \mathrm{C}$. The $\mathrm{T}_{\mathrm{m}}$ was used as the trigger temperature for shape recovery thus achieving a $\mathrm{T}_{\mathrm{m}}$ close to the human body temperature which is very desirable for material intended to be used as biomedical material. The obtained result is consistent with a previous study, where the fabricated shape memory polymer showed a similar $\mathrm{T}_{\mathrm{m}}$ around $38{ }^{\circ} \mathrm{C}$ [13].

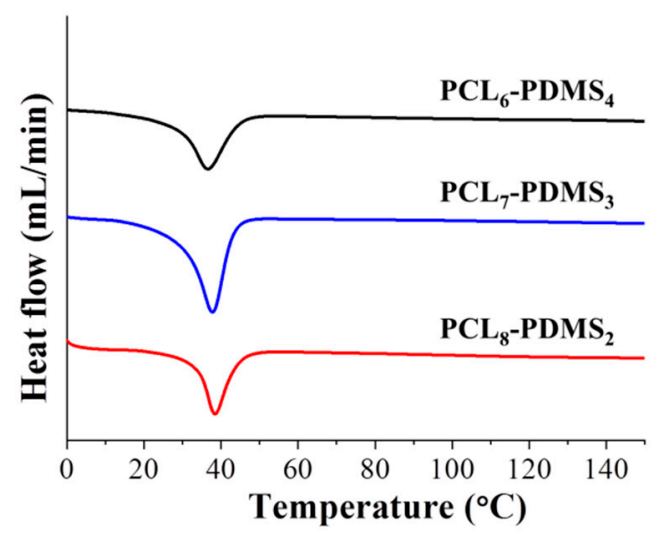

Figure 4. The differential scanning calorimetry (DSC) curves of the electro-spun PCL-PDMS copolymer nanofibres by the second heating run.

Although the PCL-PDMS mixture ratio did not display any effect on the melting temperature, however, the crystallinity $\left(\mathrm{X}_{\mathrm{c}}\right)$ and enthalpy change $\left(\Delta \mathrm{H}_{\mathrm{m}}\right)$ showed the opposite effect. Both parameters demonstrated a positive correlation to the amount of PCL segment ratio. For example, the $X_{c}$ increased from $21.93 \%$ to $26.83 \%$ when the PCL ratio increased from $60 \%\left(\mathrm{PCL}_{6}-\mathrm{PDMS}_{4}\right)$ to $80 \%\left(\mathrm{PCL}_{8}-\mathrm{PDMS}_{2}\right)$ of the copolymer ratio. Besides, the enthalpy change increased from 30.59, 34.82, and $37.42(\mathrm{~J} / \mathrm{g})$ for $\mathrm{PCL}_{6}-\mathrm{PDMS}_{4}, \mathrm{PCL}_{7}-\mathrm{PDMS}_{3}$, and $\mathrm{PCL}_{8}-\mathrm{PDMS}_{2}$, respectively. The thermal properties of the fabricated PCL-PDMS copolymer nanofibres are presented in Table 2.

Table 2. The thermal properties of the PCL-PDMS copolymer samples measured by DSC.

\begin{tabular}{cccc}
\hline Sample & $\mathbf{X}_{\mathbf{C}}(\mathbf{\%})$ & $\mathbf{T}_{\mathbf{m}}\left({ }^{\circ} \mathbf{C}\right)$ & $\Delta \mathbf{H}_{\mathbf{m}}(\mathbf{J} / \mathbf{g})$ \\
\hline PCL $_{6}-\mathrm{PDMS}_{4}$ & $21.93 \pm 2.1$ & $36.44 \pm 1.00$ & $30.59 \pm 2.9$ \\
PCL $_{7}-\mathrm{PDMS}_{3}$ & $24.96 \pm 1.9$ & $37.41 \pm 0.91$ & $34.82 \pm 2.75$ \\
$\mathrm{PCL}_{8}-\mathrm{PDMS}_{2}$ & $26.83 \pm 2.7$ & $37.70 \pm 1.12$ & $37.42 \pm 3.8$ \\
\hline
\end{tabular}

$\mathrm{T}_{\mathrm{m}}$ (melting temperature) and $\Delta \mathrm{H}_{\mathrm{m}}$ (enthalpy change) determined by the second heating run of DSC curves.

\subsection{Mechanical Properties of the PCL-PDMS Copolymer Nanofibres}

Figure 5 illustrates the stress-strain curve of the copolymer nanofibres during the uniaxial tensile test. The tensile properties of the copolymer nanofibres such as tensile strength, percent of elongation and Young's modulus are tabulated in Table 3. The tensile properties of the copolymer nanofibres were highly dependent on the copolymer mixture ratio. The tensile strength of the $\mathrm{PCL}_{8}-\mathrm{PDMS}_{2}$ is 1.41 MPa, and when the ratio of PDMS content increases to $\mathrm{PCL}_{6}-\mathrm{PDMS}_{4}$, the tensile strength increases 
to $6.64 \mathrm{MPa}$. In addition, the percent of elongation ranging between $122.66 \%$ to $154.44 \%$ and the modulus ranged from 3.42 MPa to $12.65 \mathrm{MPa}$ with the addition of the PDMS soft segment. The obtained result differed from some published studies that stated that the higher PCL content increases the crystallinity of the copolymer fibres thus increase the tensile properties of the nanofibres [3]. This result might be attributed to the fabricated nanofibre morphology where the uniformity of the diameter of the $\mathrm{PCL}_{6}-\mathrm{PDMS}_{4}$ nanofibres could distribute the stress during the tensile test, therefore, providing a more stable structure. Meanwhile, the melted fibrous structure indicating insufficient physical cross-linking with less interconnected junction formed among the fibres [14] within the $\mathrm{PCL}_{8}-\mathrm{PDMS}_{2}$ sample caused the reduction in tensile strength. Moreover, it was reported that the addition of the PDMS soft segment content improves the tensile properties of SMP due to the higher soft segment entanglement density [15]. The fact that the PDMS had low $\mathrm{T}_{\mathrm{g}}\left(-125^{\circ} \mathrm{C}\right)$ makes PDMS a particularly effective soft segment which should enhance the deformability [8].

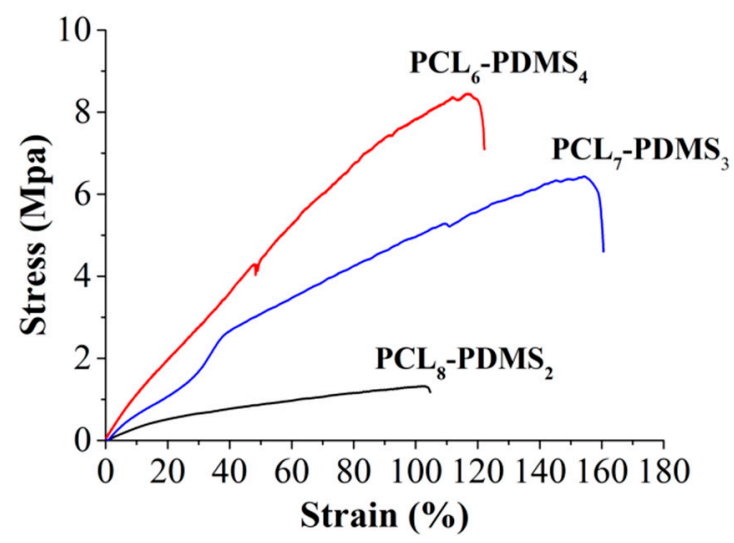

Figure 5. The stress-strain curves of the electro-spun PCL-PDMS copolymer nanofibres obtained from the tensile test.

Table 3. The mechanical properties of electro-spun PCL-PDMS copolymer nanofibres.

\begin{tabular}{cccc}
\hline Sample & Tensile Strength (MPa) & Elongation at Break (\%) & Young's Modulus (MPa) \\
\hline PCL $_{6}-\mathrm{PDMS}_{4}$ & $6.64 \pm 1.5$ & $122.66 \pm 23.3$ & $12.65 \pm 3.9$ \\
$\mathrm{PCL}_{7}-\mathrm{PDMS}_{3}$ & $4.30 \pm 1.5$ & $154.44 \pm 16.6$ & $7.93 \pm 1.0$ \\
$\mathrm{PCL}_{8}-\mathrm{PDMS}_{2}$ & $1.41 \pm 0.57$ & $137.52 \pm 24.68$ & $3.42 \pm 0.79$ \\
\hline
\end{tabular}

\subsection{The Shape Memory Behaviour}

The shape memory behaviour of the PCL-PDMS copolymer nanofibres was evaluated using thermomechanical cycles. The shape memory behaviour of the PCL-PDMS copolymer nanofibres for one complete cycle is shown in Figure 6a. The cycles involved programming the samples by heating at an elevated temperature, stretching to the desired shape at $40{ }^{\circ} \mathrm{C}$ (above $\mathrm{T}_{\mathrm{m}}$ ) until the shape was fixed and cooling to a temperature lower than $\mathrm{T}_{\mathrm{m}}\left(25^{\circ} \mathrm{C}\right)$ so that the chain segment of the samples was in a temporary position before the stress was completely removed. The samples were then re-heated to $40^{\circ} \mathrm{C}$, relaxation occurred, and the temporarily deformed samples recovered to their original permanent shape. The time taken for complete shape recovery of the sample was also recorded. According to Figure 6a, it was observed that the recovery process started immediately after re-heating and achieved full recovery at approximately $10 \mathrm{~s}$. Such a fast shape recovery of nanofibres in comparison to the film was achieved, due to the high surface area to volume ratio of nanofibres ensuring faster thermal conduction. [16]. The strain profiles of the five thermomechanical cycles are presented in Figure 6b. The shape memory performance in terms of shape fixity and recovery ratio for the PCL-PDMS copolymer nanofibres during thermomechanical cycles is tabulated in Table 4. 


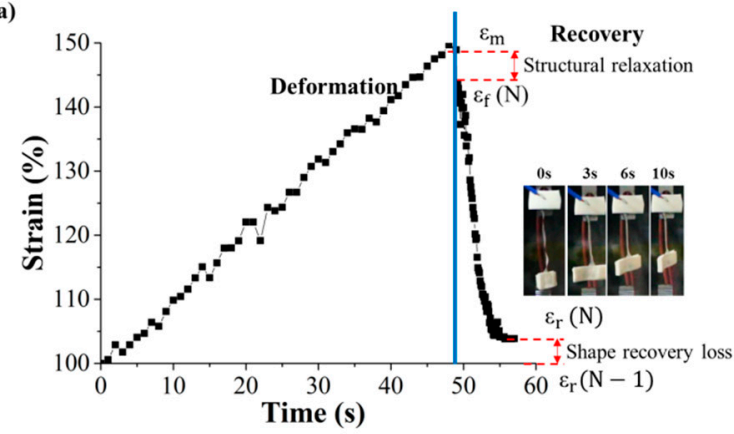

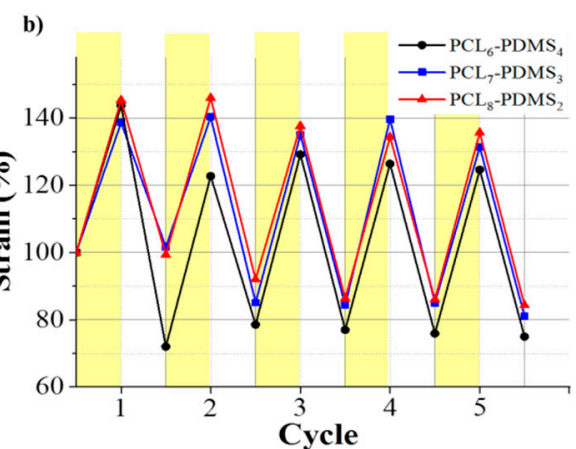

Figure 6. The shape memory behaviour of PCL-PDMS copolymer nanofibres: (a) one complete cycle as a function of time; (b) strain profile in five thermomechanical cycles.

Table 4. Shape recovery ratio $\left(R_{r}\right)$ and shape fixity ratio $\left(R_{f}\right)$ of the PCL-PDMS copolymer nanofibres for five thermomechanical cyclic tests.

\begin{tabular}{ccccccc}
\hline Sample & & Cycle 1 & Cycle 2 & Cycle 3 & Cycle 4 & Cycle 5 \\
\hline \multirow{2}{*}{ PCL $_{6}-$ PDMS $_{4}$} & $\mathrm{R}_{\mathrm{f}}(\%)$ & 86.97 & 90.18 & 91.50 & 95.78 & 98.74 \\
& $\mathrm{R}_{\mathrm{r}}(\%)$ & 163.41 & 84.43 & 103.10 & 102.16 & 101.36 \\
PCL $_{7}-\mathrm{PDMS}_{3}$ & $\mathrm{R}_{\mathrm{f}}(\%)$ & 90.14 & 95.49 & 85.97 & 94.27 & 93.52 \\
& $\mathrm{R}_{\mathrm{r}}(\%)$ & 95.69 & 142.93 & 101.46 & 98.79 & 110.99 \\
PCL $_{8}-\mathrm{PDMS}_{2}$ & $\mathrm{R}_{\mathrm{f}}(\%)$ & 83.72 & 90.87 & 92.49 & 95.53 & 96.07 \\
& $\mathrm{R}_{\mathrm{r}}(\%)$ & 101.47 & 115.71 & 112.41 & 100.69 & 100.69 \\
\hline
\end{tabular}

The PCL-PDMS copolymer nanofibres exhibited excellent shape fixity ratio which was above $83 \%$ for the first tensile cycle and exceeded $90 \%$ for subsequent cycles. The shape fixity of the copolymer nanofibres was lower in the first cycle because of the segment-chain orientation and relaxation effects $[17,18]$. As such, the shape memory nanofibres should be pre-conditioned to fully utilise the shape memory potential $[13,17,18]$. For subsequent cycles, the shape fixity of all samples surpassed $90 \%$. After the fifth cycle, all samples maintained an excellent shape fixity performance up to $98 \%$ for $\mathrm{PCL}_{6}-\mathrm{PDMS}_{4}$. The samples were capable of maintaining the deformed shape and were not weakened regardless of the copolymer mixture ratio as proven by the excellent fixity ratio. The excellent fixity shown by the copolymer PCL-PDMS nanofibres was associated with the physical cross-links formed through inter- or intra-polymeric chain attractions such as hydrogen bonding or dipole-dipole interaction. Moreover, the degree of crystallinity for all samples obtained from the DSC was higher than 20\% (Table 2) which allows for a high strain fixity ratio [19]. On the other hand, the shape recovery ratio for all samples was higher than $100 \%$.

Our result shows the recovery ratio exceeded $100 \%$ which means that the length of the recovered SMP after heating was shorter than the original permanent shape. This might be due to the super-contraction behaviour of the nanofibre. The pre-orientation of the nanofibre during the electrospinning process leads to the super-contraction behaviour. At the temporary state, the nanofibres were pre-stretched causes a certain degree of molecular orientation and electrostatic repulsion. When the nanofibre was heated to recover the permanent shape, thermal expansion induced re-crystallization of the PCL. Rapid crystallization of PCL segments during the cooling process formed a more compact structure of the fibre [20]. The thermal expansion effect is neglected in the discussion. Besides, the shape recovery $>100 \%$ were reported in the previous reports for PCL-PDMS SMP [18,20]. The super-contraction behaviour of the SMP helps to maintain the shape after several cycles and prevent the fibre from sagging. In one report, the super-contraction behaviour of SMP helps to heal macroscopic damages as the fibres exert a higher compressive force on the damage interfaces due to the tendency to shrink further [16]. Since the thermomechanical cycles are repeatable, therefore, the capability to heal macroscopic damages may be repeatable. The $\mathrm{PCL}_{6}-\mathrm{PDMS}_{4}$ nanofibres with the largest diameter and amorphous domain yield the highest shape recovery in the first cycle (163.41\%). 
Based on the result, it can be concluded that both the shape fixity and recovery ratio was independent of the number of cycles.

Furthermore, the nanofibres' surface morphology during deformation and fixation after shape recovery was assessed, and the SEM images are presented in Figure 7. Different nanofibres structures are observed before and after the deformation. Initially, the fibres show a randomly oriented structure (Figure 3); however, after the temporary stretch (Figure 7a-c), the fibres tend to align along the strain direction. The samples maintained continuity and no broken fibres were detected, indicating the flexibility of the samples. Once the samples were re-heated, the directed nanofibres recovered to their original random orientation. The average nanofibres' diameter increased in comparison to the initial state (Figure 3) after temporary deformation with the sample $\mathrm{PCL}_{6}-\mathrm{PDMS}_{4}$ showing the highest diameter increase from $348 \mathrm{~nm}$ to $1048 \mathrm{~nm}$. Following the shape recovery, the average fibre diameter recovered to around $1143 \mathrm{~nm}, 1152 \mathrm{~nm}$ and $654 \mathrm{~nm}$ for $\mathrm{PCL}_{6}-\mathrm{PDMS}_{4}, \mathrm{PCL}_{7}-\mathrm{PDMS}_{3}$ and $\mathrm{PCL}_{8}-\mathrm{PDMS}_{2}$, respectively.
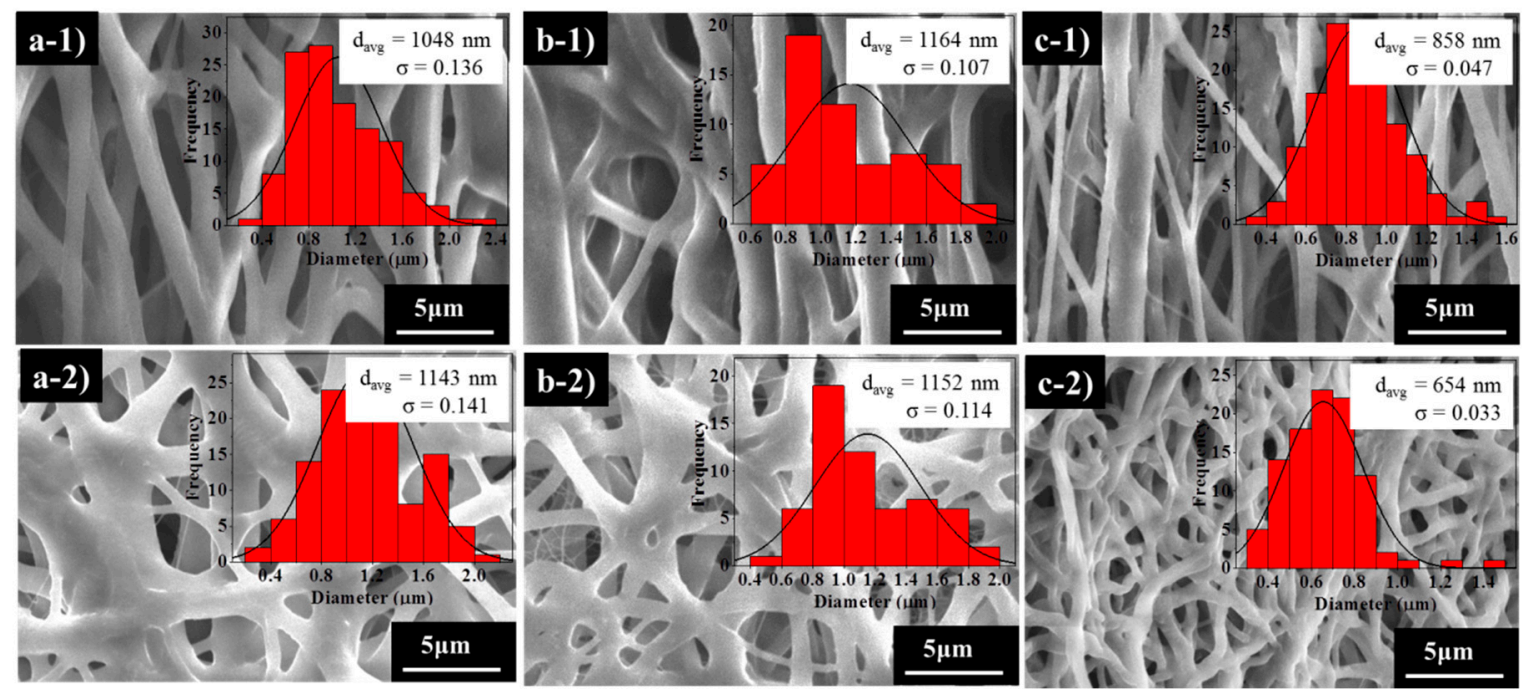

Figure 7. The SEM images of the nanofibres' structure during the deformation and after recovery: (a-1,a-2) $\mathrm{PCL}_{6}-\mathrm{PDMS}_{4} ;(\mathbf{b}-\mathbf{1}, \mathbf{b}-\mathbf{2}) \mathrm{PCL}_{7}-\mathrm{PDMS}_{3} ;(\mathbf{c}-\mathbf{1}, \mathbf{c}-\mathbf{2}) \mathrm{PCL}_{8}-\mathrm{PDMS}_{2}$.

\subsection{Cytotoxicity and Biocompatibility Study}

The cytotoxicity of the fabricated nanofibres was statistically evaluated using the CCK-8 assay to validate their application in the biomedical field. Human dermal fibroblast cells were cultured on the fabricated nanofibres and the cytotoxicity effect was observed. The HDF cells cultured on a tissue culture plate (TCP) were regarded as the control group. Figure 8 shows the CCK- 8 result for all samples after $24 \mathrm{~h}$ and $72 \mathrm{~h}$. The viability is presented in terms of relative fold change from the control group.

The HDF cell viability is presented in terms of relative fold change from the control group. As expected, since the copolymer samples are based on derivatives of biocompatible PCL and PDMS, no samples showed any toxicity effect to the HDF cells and, as such, this demonstrates compatibility for biomedical application. According to Figure 8, the cellular viability for all samples increased significantly compared to the control group after $72 \mathrm{~h}$, assuming that the nanofibres can promote cell growth and proliferation. This confirms that the fabricated copolymer nanofibre did not exhibit any toxicity to the cells for $72 \mathrm{~h}$.

Although the fabricated nanofibres showed low cell viability percentage, this shrinkable nanofibre mesh can be applied as an early treatment on acute open wounded area during an emergency situation. Since the fabricated SMP was triggered at near body temperature $\left(37^{\circ} \mathrm{C}\right)$, the pre-stretched fibre mesh can recover to its initial shape upon applying to body. The shrinkage of the fibre during the shape recovery gives pressure to the wound site. 
Besides, HDF cells seeded on the nanofibre samples were stained with FDA/DAPI double staining for observable evaluation on the cell morphology. The microscopy images of the cell morphology illustrated in Figure 9. Survival cells were observed in all samples with spreading cellular extensions.

a)

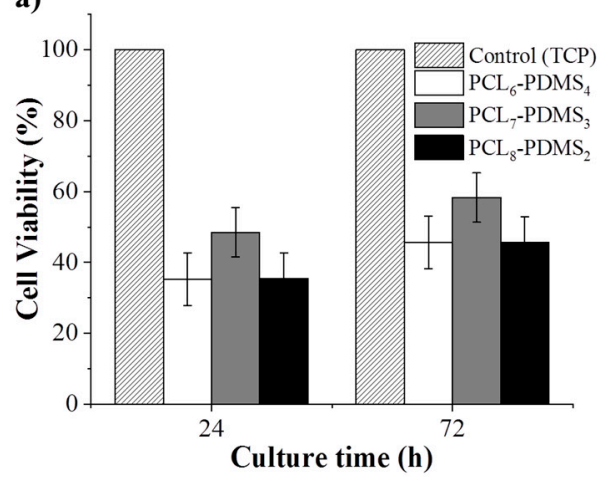

b)

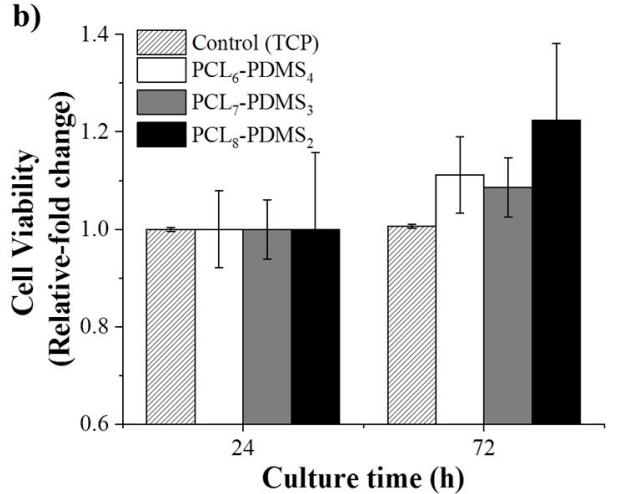

Figure 8. The biocompatibility of PCL-PDMS nanofiber mesh based on (a) viability percentage and (b) relative fold change relative to the control group.
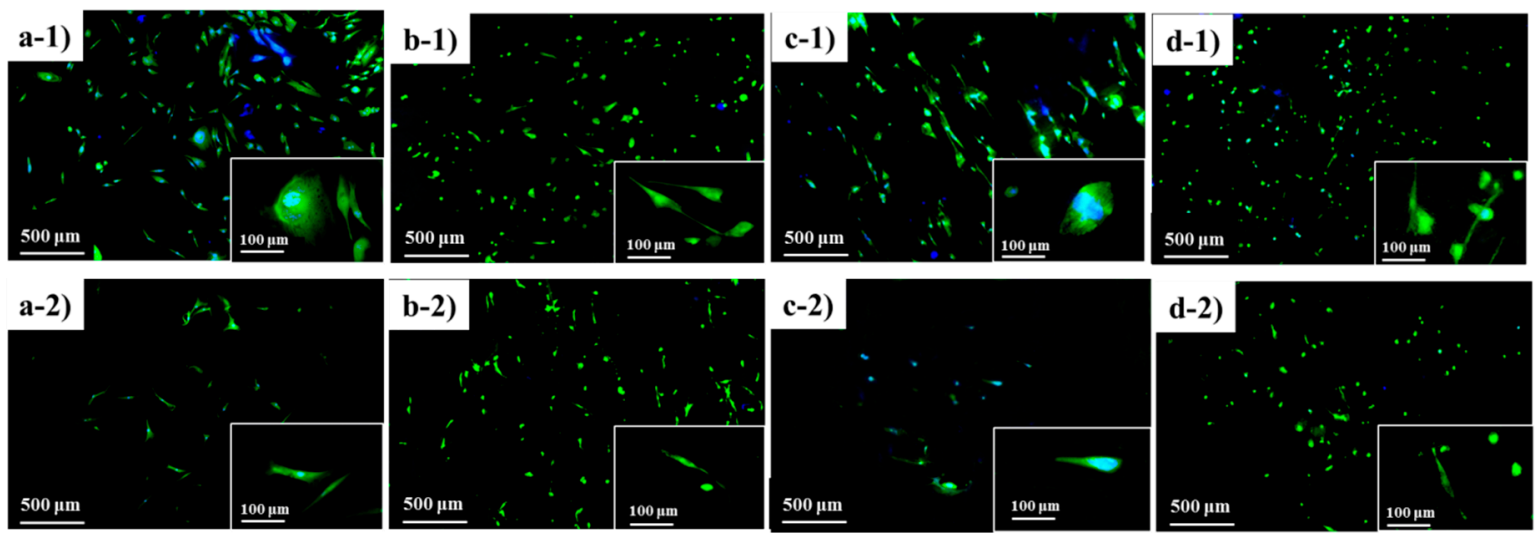

Figure 9. Dual staining of fluorescein diacetate-4',6-diamidino-2-phenylindole (FDA/DAPI) for biocompatibility study: (a-1,a-2) control group (TCP); (b-1,b-2) $\mathrm{PCL}_{6}-\mathrm{PDMS}_{4}$; (c-1,c-2) $\mathrm{PCL}_{7}-\mathrm{PDMS}_{3}$; and (d-1,d-2) $\mathrm{PCL}_{8}-\mathrm{PDMS}_{2}$.

\subsection{Demonstration of the Shape Memory Response}

The images in Figure 10 demonstrate the shape memory responses of the strip made up by electro-spun $\mathrm{PCL}_{6}-\mathrm{PDMS}_{4}$ nanofibres. To perform a visualized demonstration of shape memory responses, we wrapped a sponge with the PCL-PDMS strip which was programmed as a temporary shape state. Before that, the PCL-PDMS strip with $30 \mathrm{~mm}$ in length was, initially, stretched to $50 \%$ strain at $40{ }^{\circ} \mathrm{C}$ by using the tensile machine under the elongation speed of $20 \mathrm{~mm} / \mathrm{min}$. The strip was kept at $45 \mathrm{~mm}$ as a temporary shape state on the stage until the environmental temperature reached room temperature. As presented in Figure 10b, the ambient environment was heated again to $40{ }^{\circ} \mathrm{C}$, and the stretched nanofibres shrunk to its initial length of $30 \mathrm{~mm}$ within $10 \mathrm{~s}$ as demonstrated in Figure 10d,e. Figure 10f,g showed the photos of nanofibre mesh which was cut into the size of $10 \mathrm{~mm} \times 10 \mathrm{~mm}$. Nanofibre mesh had undergone a series of temperature changes to demonstrate its shape memory ability. The nanofibre mesh was stretched to $200 \%$ at $40{ }^{\circ} \mathrm{C}$ (Figure $10(\mathrm{f}-1)$ ). After that, the nanofibre mesh was cooled to $25{ }^{\circ} \mathrm{C}$ until equilibrium was reached. Lastly, we could observe that after reheating nanofibre mesh to $40{ }^{\circ} \mathrm{C}$, the nanofibre mesh recovered to the permanent shape (Figure 10(g-1)). As presented in the SEM image of Figure 10(f-2,g-2), we could conclude that the microstructure of nanofibre mesh changed with the shape of nanofibre mesh. The fibres showed the arranged orientation after being stretched (Figure 10(f-2)). Meanwhile, the energy generated by stretching was stored in the deformed nanofibre mesh. The increase in temperature caused the entropy 
change which, in turn, leads to changes in the macroscopic appearance. The fibre reverted to random orientation again (Figure 10(g-2)).

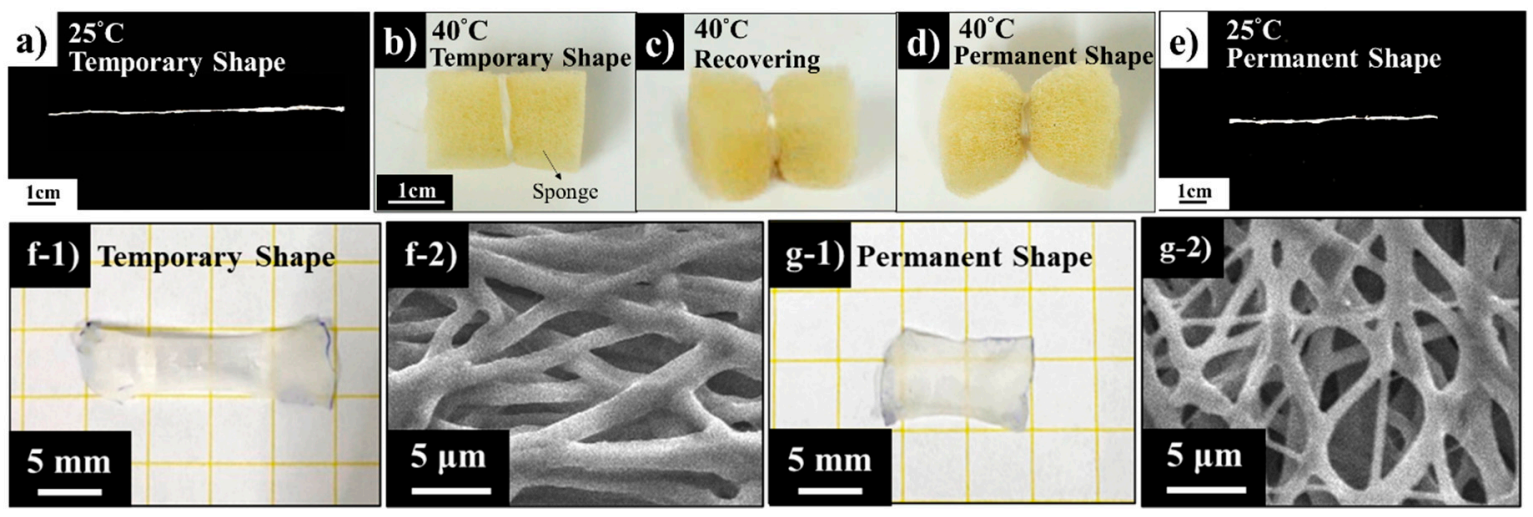

Figure 10. Visual demonstration of the nanofibres' shape recovery performance: $(\mathbf{a}, \mathbf{b})$ temporary shape; (c) during recovery; (d) recovered to the permanent shape; (e) permanent shape; (f-1) temporary shape macroscopic view of nanofibre mesh; (f-2) temporary shape microscopic view of nanofibre mesh; (g-1) permanent shape macroscopic view of nanofibre mesh; (g-2) permanent shape microscopic view of nanofibre mesh.

\section{Conclusions}

We fabricated and characterised polycaprolactone-based shape memory polymer (SMP) with different polydimethylsiloxane (PDMS) soft-segment mixture ratios. Depending on the PDMS soft-segment content, a series of PCL-PDMS copolymers, namely, $\mathrm{PCL}_{6}-\mathrm{PDMS}_{4}, \mathrm{PCL}_{7}-\mathrm{PDSM}_{3}$ and $\mathrm{PCL}_{8}-\mathrm{PDMS}_{2}$ fibres, were fabricated and engineered to nanofibre mesh structure with nanofibre diameter ranging from $151 \mathrm{~nm}$ to $622 \mathrm{~nm}$. The fabricated PCL-PDMS copolymer can perform the shape changes with a large deformation triggered by temperature at approximately $37^{\circ} \mathrm{C}$. The mechanical properties of the PCL-PDMS copolymer fibres improve with the addition of PDMS soft segment. By comparing with the $\mathrm{PCL}_{7}-\mathrm{PDSM}_{3}$ and $\mathrm{PCL}_{8}-\mathrm{PDMS}_{2}$ fibre samples, $\mathrm{PCL}_{6}-\mathrm{PDMS}_{4}$ possessed the highest tensile properties with 6.64 MPa tensile strength and elongation of up to $122.7 \%$. After five thermomechanical cycles, all PCL-PDMS fibre samples exhibited excellent shape recovery and shape fixity which was around 98\% and 100\%, respectively. The PCL-PDMS fibres demonstrated a fast response and required around $10 \mathrm{~s}$ to completely recover its original shape. Based on the experimental results, including appropriate mechanical properties, biocompatibility, along with excellent shape memory behaviour, we believe that the fabricated PCL-PDMS copolymer nanofibre mesh has great potential to be applied in biomedical application such as for shrinkable tubing and surgical suture.

Author Contributions: Conceptualization, W.-C.L. and Y.K.; experiments and data analysis, C.-H.H., N.A.M.R., Z.-W.Y., W.-C.L. and Y.K.; writing and editing, N.A.M.R., C.-H.H. and W.-C.L.; photos and figure preparation, N.A.M.R., C.-H.H., Z.-W.Y. and D.I.; supervision, W.-C.L., and Y.K.; funding acquisition, W.-C.L. and H.-H.S. All authors have read and agreed to the published version of the manuscript.

Funding: This study was supported by a research grant from the Ministry of Science and Technology (MOST108-2221-E-110-066), Ministry of Education (1070009000), and Kaohsiung Veterans General Hospital (VGHNSU109-005). The authors gratefully acknowledge the assistance, facilities, and support supply from the NSYSU-Biomimicking \& Engineering Lab (Being ${ }^{2}$ Lab) National Sun Yat-sen University, Kaohsiung, Taiwan.

Conflicts of Interest: The authors declare no conflicts of interest.

\section{References}

1. Lendlein, A.; Langer, R. Biodegradable, elastic shape-memory polymers for potential biomedical applications. Science 2002, 296, 1673-1676. [CrossRef] [PubMed]

2. Yakacki, C.M.; Gall, K. Shape-memory polymers for biomedical applications. In Shape-Memory Polymers; Springer: Berlin, Germany, 2009; pp. 147-175. 
3. Zhang, D.; Giese, M.L.; Prukop, S.L.; Grunlan, M.A. Poly ( $\varepsilon$-caprolactone)-based shape memory polymers with variable polydimethylsiloxane soft segment lengths. J. Polym. Sci. Part A Polym. Chem. 2011, 49, 754-761. [CrossRef] [PubMed]

4. Luo, X.; Mather, P.T. Preparation and characterization of shape memory elastomeric composites. Macromolecules 2009, 42, 7251-7253. [CrossRef]

5. Wache, H.; Tartakowska, D.; Hentrich, A.; Wagner, M. Development of a polymer stent with shape memory effect as a drug delivery system. J. Mater. Sci. Mater. Med. 2003, 14, 109-112. [CrossRef] [PubMed]

6. Yakacki, C.M.; Shandas, R.; Lanning, C.; Rech, B.; Eckstein, A.; Gall, K. Unconstrained recovery characterization of shape-memory polymer networks for cardiovascular applications. Biomaterials 2007, 28, 2255-2263. [CrossRef] [PubMed]

7. Udomluck, N.; Koh, W.-G.; Lim, D.-J.; Park, H. Recent Developments in Nanofiber Fabrication and Modification for Bone Tissue Engineering. Int. J. Mol. Sci. 2020, 21, 99. [CrossRef] [PubMed]

8. Schoener, C.A.; Weyand, C.B.; Murthy, R.; Grunlan, M.A. Shape memory polymers with silicon-containing segments. J. Mater. Chem. 2010, 20, 1787-1793. [CrossRef]

9. Mei, N.; Chen, G.; Zhou, P.; Chen, X.; Shao, Z.-Z.; Pan, L.-F.; Wu, C.-G. Biocompatibility of poly ( $\varepsilon$-caprolactone) scaffold modified by chitosan—the fibroblasts proliferation in vitro. J. Biomater. Appl. 2005, 19, 323-339. [CrossRef] [PubMed]

10. Dou, Q.Q.; Liow, S.S.; Ye, E.; Lakshminarayanan, R.; Loh, X.J. Biodegradable thermogelling polymers: working towards clinical applications. Adv. Healthc. Mater. 2014, 3, 977-988. [CrossRef]

11. Crescenzi, V.; Manzini, G.; Calzolari, G.; Borri, C. Thermodynamics of fusion of poly- $\beta$-propiolactone and poly- $\epsilon$-caprolactone. comparative analysis of the melting of aliphatic polylactone and polyester chains. Eur. Polym. J. 1972, 8, 449-463. [CrossRef]

12. Xie, T. Tunable polymer multi-shape memory effect. Nature 2010, 464, 267-270. [CrossRef] [PubMed]

13. Chan, B.Q.Y.; Liow, S.S.; Loh, X.J. Organic-inorganic shape memory thermoplastic polyurethane based on polycaprolactone and polydimethylsiloxane. Rsc Adv. 2016, 6, 34946-34954. [CrossRef]

14. Kai, D.; Prabhakaran, M.P.; Chan, B.Q.Y.; Liow, S.S.; Ramakrishna, S.; Xu, F.; Loh, X.J. Elastic poly ( $\varepsilon$-caprolactone)-polydimethylsiloxane copolymer fibers with shape memory effect for bone tissue engineering. Biomed. Mater. 2016, 11, 015007. [CrossRef] [PubMed]

15. John, J.V.; Moon, B.K.; Kim, I. Influence of soft segment content and chain length on the physical properties of poly (ether ester) elastomers and fabrication of honeycomb pattern and electrospun fiber. React. Funct. Polym. 2013, 73, 1213-1222. [CrossRef]

16. Li, G.; Meng, H.; Hu, J. Healable thermoset polymer composite embedded with stimuli-responsive fibres. J. R. Soc. Interface 2012, 9, 3279-3287. [CrossRef] [PubMed]

17. Zhang, Y.; Wang, Q.; Wang, C.; Wang, T. High-strain shape memory polymer networks crosslinked by $\mathrm{SiO}_{2}$. J. Mater. Chem. 2011, 21, 9073-9078. [CrossRef]

18. Zhang, D.; Petersen, K.M.; Grunlan, M.A. Inorganic-organic shape memory polymer (SMP) foams with highly tunable properties. Acs Appl. Mater. Interfaces 2013, 5, 186-191. [CrossRef] [PubMed]

19. Lu, X.; Sun, Z.; Cai, W.; Gao, Z. Study on the shape memory effects of poly (l-lactide-co- $\varepsilon$-caprolactone) biodegradable polymers. J. Mater. Sci. Mater. Med. 2008, 19, 395-399. [CrossRef] [PubMed]

20. Zhang, D.; Burkes, W.L.; Schoener, C.A.; Grunlan, M.A. Porous inorganic-organic shape memory polymers. Polymer 2012, 53, 2935-2941. [CrossRef] [PubMed]

(C) 2020 by the authors. Licensee MDPI, Basel, Switzerland. This article is an open access article distributed under the terms and conditions of the Creative Commons Attribution (CC BY) license (http://creativecommons.org/licenses/by/4.0/). 